\title{
Age-related features of stress tolerance of the nervous system
}

\author{
Anna Ivanova*, Anna Korban, Nikolay Ryzhkin, and Yulia Savchenko \\ Don State Technical University, 344002, Rostov-on-Don, Russia
}

\begin{abstract}
The article is devoted to the study of age-related features of the display of varying degrees of courage and stress tolerance to a dangerous situation that cannot be predicted, created in the course of the experiment, as close as possible to the real situation. Each factor of the typological complex was evaluated by a relation coefficient in the system. In comparing the age-related features of the display of courage with the agerelated features of the display of the main characteristics of the nervous system, it was found that the latter change in such a way that in one age period they contribute to the display of courage, but in another age period, on the contrary, they prevent the display of courage. The comparison of age-related change in the number of persons with extinction mobility with a high degree of courage shows that in many age groups they change in different directions, but this divergence indicates that the change in the typological feature corresponds to the nature of the change in the degree of courage.
\end{abstract}

\section{Introduction}

The increasing competition in the international sporting arena makes it necessary to start high-level athletes' training from their early pre-school age. During doing various sports disciplines, there are situations that give rise to the developing a sportsman's strong feeling of fear. In this regard, one of the qualities that determine the success of progression in sports is "courage". There is little information on the subject of athletes' stress tolerance and fears in various literary sources and on the Internet. The available fragmented knowledge does not represent the full picture of the issue.

\section{Materials and methods}

The study was conducted on the basis of Don State Technical University (Rostov-on-Don) on three groups of subjects between the age of 8 to 26 :

1) on students of the lyceum at DSTU who are engaged in physical education within the content of school curriculum;

2) on volleyball players of specialized children's and youth sports school;

3) on students of Institute of Physical Culture and Sports at DSTU.

\footnotetext{
${ }^{*}$ Corresponding author: an.badm@yandex.ru
} 
There were studied the age-related features of the display of varying degrees of courage and stress tolerance to a dangerous situation that cannot be predicted, created in the course of the experiment, as close as possible to the real situation.

All the subjects went through two tests of the experiment.

The first test is reverse straddle vault over a bar set at a height equal to one third of the subject's height. Only one attempt was given for doing the exercise. The vault was done once with open eyes, and the other time with closed eyes (a special blindfold was used).

The second test is squat vault over a bar, which height was three quarters of the subject's own height. The vault was done with and without safeguarding.

The scores, that the subjects received for doing exercises with open and closed eyes were compared with each other. This was the criterion for dividing the subjects into experimental groups with a high, medium and low degree of courage, as can be seen from figure 1. The first graph shows male persons, and the second graph, respectively, shows female persons.
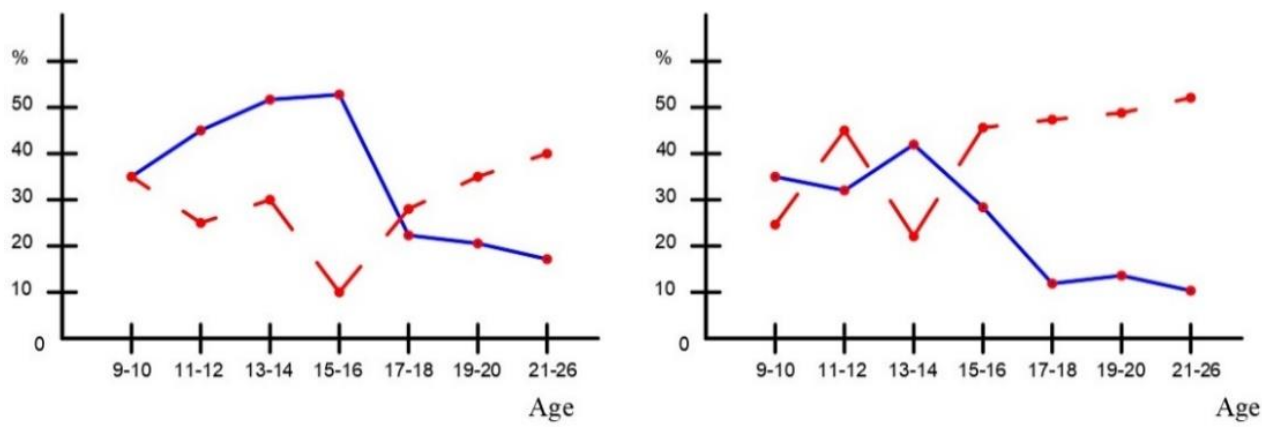

Fig. 1. Age-related changes in the number of persons with high and low levels of courage

The group with a high degree of courage included the subjects who, during doing exercises in a dangerous situation (with closed eyes and without safeguarding), did not impair their quality compared to doing the exercise in less stringent conditions (with open eyes and with safeguarding), in other words, when the scores were compared, these subjects received a positive difference or a difference equal to zero. The group with a low degree of courage included the subjects who had impaired the quality of doing both exercises in a dangerous situation, or rather, received a negative difference. The group with an average degree of courage included the subjects who, during doing one of the two exercises, overcame a dangerous situation (did not impair but even improve the quality of doing the exercise), and during doing the other exercise, impaired the quality of doing.

In order to prevent the influence of exterior irritator in the process of conducting the experiment, only 1-2 people were invited for the first test, and 2-3 people for the second one.

The number of persons assigned to a particular group, depending on the degree of "courage" was calculated at each age. The comparison of changes in the numerical composition of these groups because of age was drawn separately for male and female persons.

\section{Results}

To compare these groups, the numerical composition of persons was expressed as a percentage (the total number of persons who had taken part in the experiment in a certain age and gender group was taken as $100 \%$ ). The results can be seen in tables 1 and 2 . 
Table 1. Age-related changes in the number of male persons with varying degrees of courage

\begin{tabular}{|c|c|c|c|c|c|c|c|c|}
\hline \multicolumn{2}{|c|}{ Age } & $9-10$ & $11-12$ & $13-14$ & $15-16$ & $17-18$ & $19-20$ & $21-26$ \\
\hline \multicolumn{2}{|c|}{ Number of subjects } & 59 & 56 & 88 & 67 & 46 & 37 & 44 \\
\hline $\begin{array}{c}\text { Percentage } \\
\text { of persons } \\
\text { with varying } \\
\text { degrees of } \\
\text { courage }\end{array}$ & High & 35.5 & 40.3 & 51 & 53 & 22 & 22 & 20 \\
\cline { 2 - 9 } & Low & 35.5 & 27 & 31.1 & 11 & 29.5 & 34 & 38 \\
\hline
\end{tabular}

Table 2. Age-related changes in the number of female persons with varying degrees of courage

\begin{tabular}{|c|c|c|c|c|c|c|c|c|c|c|}
\hline \multicolumn{2}{|c|}{ Age } & \multicolumn{2}{|c|}{$9-10$} & \multicolumn{2}{|c|}{$11-12$} & \multirow[t]{2}{*}{$13-14$} & \multirow[t]{2}{*}{$15-16$} & \multirow[t]{2}{*}{$17-18$} & \multirow[t]{2}{*}{$19-20$} & \multirow[t]{2}{*}{$21-26$} \\
\hline & & 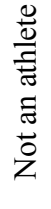 & 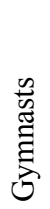 & 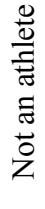 & 苟 & & & & & \\
\hline \multicolumn{2}{|c|}{ Number of subjects } & 69 & 39 & 64 & 32 & 58 & 77 & 37 & 19 & 24 \\
\hline \multirow{3}{*}{$\begin{array}{c}\text { Percentage } \\
\text { of persons } \\
\text { with } \\
\text { varying } \\
\text { degrees of } \\
\text { courage }\end{array}$} & High & 33 & 31 & 28 & 27,3 & 41.8 & 28.2 & 13.4 & 13 & 11.8 \\
\hline & $\begin{array}{c}\text { Mediu } \\
\mathrm{m}\end{array}$ & 38 & 35 & 24 & 34.7 & 33.9 & 25.6 & 41.4 & 40 & 37 \\
\hline & Low & 29 & 34 & 48 & 38 & 24.3 & 46. & 45.2 & 47 & 51.2 \\
\hline
\end{tabular}

The picture of age-related changes in the number of persons with a low degree of courage is reversed. The lowest number (29\%) of them is at $13-14$ years, then it increases to $45.2 \%$ at the age of $17-18$ years and to $51.2 \%$ in the subsequent years.

If we compare the age-related changes of courage among male and female persons, it should be noted that the increase in the number of persons with a high degree of courage begins both among women and men from the age of 11 to 12, but the increase in the number of brave persons among women stops earlier than among men. Before adulthood, both men and women (10-11 years old) have an almost equal number of those assigned to the group with a high degree of courage. With the end of sexual development, the number of persons assigned to the group with a high degree of courage among the representatives of the female subjects, is almost twice as little than among the representatives of the male subjects. These features of the display of courage at the age of 11-14 years among female representatives and at the age of 11-16 years among male representatives, obviously, should be associated with an increasing influence of the endocrine system during adulthood, which has a strong influence on the mental regulation of human activity.

It is interesting to note that young female athletes who are engaged in sports colleges do not differ from those engaged in physical education within the school curriculum in terms of courage. So, among the female athletes aged 9-10 and 11-12 years there were found even fewer brave athletes, and there are even more volleyball players with a low degree of courage at the age of 9-10 years. However, among the female athletes aged 11-12 years, the number of persons with a low degree of courage is $10 \%$ less than among girls who are engaged in physical education within the school curriculum. Obviously, this can be explained by a more careful selection of students in sports colleges and by the fact that only girls who are characterized by courage express a desire to do sports. 


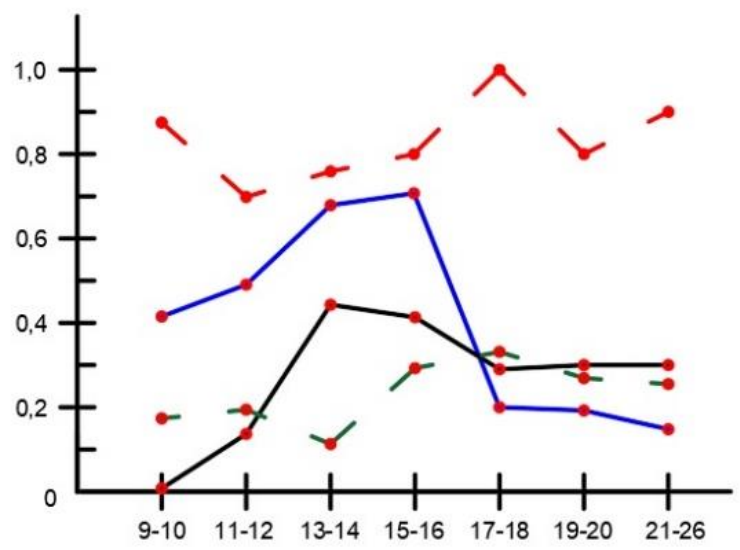

Fig. 2. Comparison of the age-related changes in the number of persons with a high degree of courage with age-related features of the display of the main characteristics of the nervous system among male persons

While we were making this comparison, it was suggested that the more persons with a weak nervous system will be at a certain age, the more persons with a low degree of courage will also be at that age.

The statistics in figures 2, 3, and 4 show that this suggestion was not in vain. Thus, among male persons, the nature of age-related changes in the number of persons with a low degree of courage corresponds to the nature of age-related changes in the number of persons with a weak nervous system in all age groups, up to 17-18 years. At the age of 1516 years, when the number of brave persons is the greatest, there is also a maximum number of male persons with a strong nervous system.

However, there are also conflicting changes in the courage and strength of the nervous system. At the age of 13-14 years, when the number of persons assigned to the group with a high degree of courage increases, the number of persons with a strong nervous system decreases. Especially many divergences in the direction of changes in the courage and strength of the nervous system are found among female persons. At the age of 11-12 years and at 15 years and older, the number of persons with a high degree of courage decreases, and the number of persons with a strong nervous system increases.

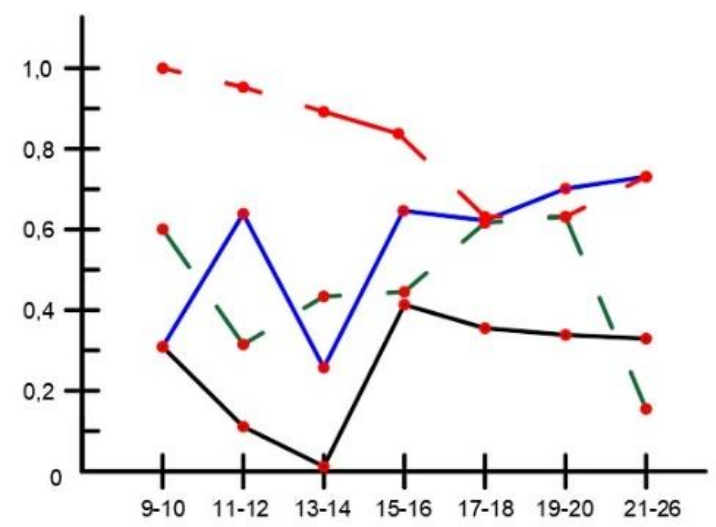

Fig. 3. Comparison of the age-related changes in the number of persons with a low degree of courage with age-related features of the display of the main characteristics of the nervous system among female persons 
The same divergences and similarities in the dynamics of age-related changes in the number of male and female persons with a low degree of courage and a weak nervous system are seen in figures 2 and 3.

It has been repeatedly stated in this work that the predominance of external excitation over extinction is characteristic for the persons with a high degree of courage, and the predominance of external extinction is characteristic for the persons with a low degree of courage. When comparing these two factors in the age aspect, their close connection is in clear view: age-related increase in the number of persons with a high degree of courage (among boys in the period of 11-16 years, among girls in the period of 13-14 years) is accompanied by an increase in the number of persons with a predominance of the excitation process.

Accordingly, during periods when the number of persons with a high degree of courage decreases, the number of persons with a predominance of extinction over excitation increases.

\section{Discussions}

The building of courage and determination plays a big part in the character-building. These qualities can be built in training and physical education classes, since the training process itself contributes to modeling all sorts of situations for this. Courage can be assessed not only by the stability of the athletic skill, which, theoretically speaking, contains elements of some risk, but also by the optimal display of physical qualities.

\section{Conclusions}

To sum up the results of comparing the age-related features of the display of courage with the age-related features of the display of the main characteristics of the nervous system, it should be noted that the latter change in such a way that in some age periods they contribute to the display of courage, but in other age periods, on the contrary, they prevent the display of courage. At the same time, these increasing and weakening displays of courage and influence of different characteristics of nervous system are not synchronous. So, while the change in the balance between external excitation and extinction in the period from 11-12 years of age to 10-16 years of age contributes to the display of courage, the strength of the nervous system in this age period decreases, which prevents the display of courage. Since the display of courage is influenced by a complex of characteristics of the nervous system (the strength of excitation, mobility, block of extinction, the balance between external excitation), it was necessary to calculate the value of the typological complex that contributes to the display of courage, and the value of the typological complex that prevents the display of courage. The calculated values of complexes in different age groups could then be compared with the already considered graph of the display of great courage and the graph of the display of small courage.

Each factor of the typological complex (for example, the greater strength of the nervous system, or the block of extinction, or the predominance of external excitation in the complex that contributes to the display of courage) was assessed by a relation coefficient in the system. First, there were calculated the interspecific relations, where the level of significance of each factor contributing to the display of a high or low degree of courage was established. Then, the complexes were averaged according to the calculated interspecific coefficients to identify the total interrelation of positive and negative complexes with the display of a high and low degree of courage. 

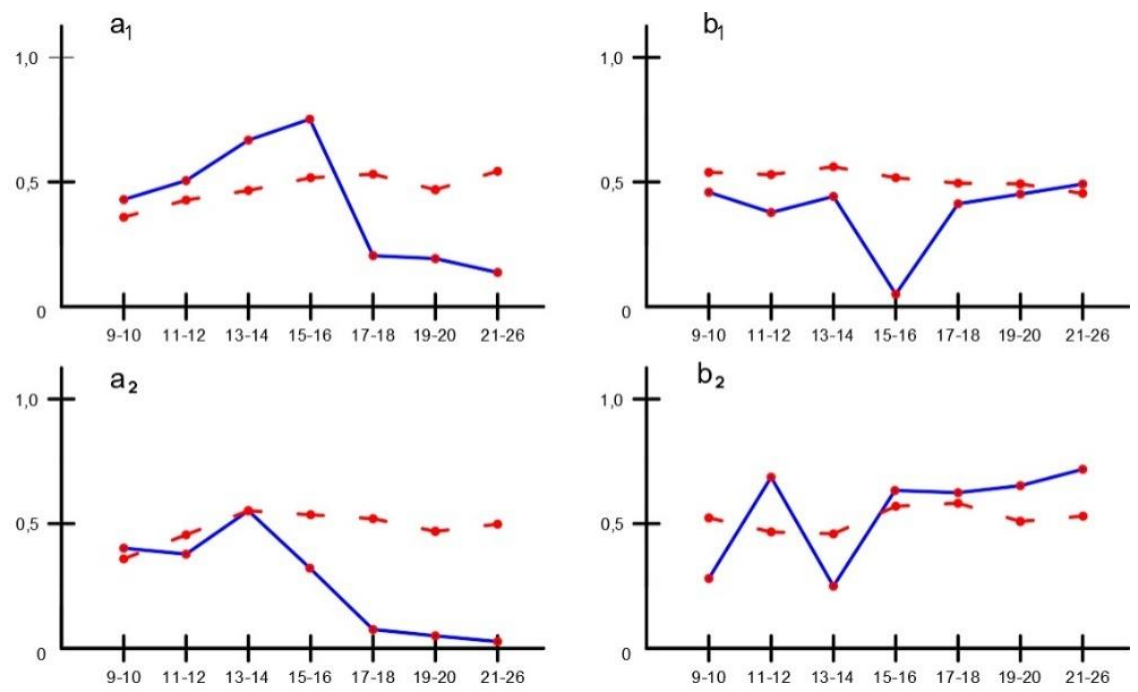

Fig. 4. Comparison of age-related changes of varying degrees of courage with age-related changes of the typological complex of courage and fearfulness

The figure 4al shows the age dynamics of the complex of high degree of courage among male persons. The nature of the change of the graph indicates the connection of a high degree of courage with a complex of factors that contribute to its display. The figure $4 \mathrm{~b} 1$ shows the age-related dynamics of the change of the low degree of courage and its comparison with the age-related change of the "cowardice" symptom-complex.

The relation between a low degree of courage and the symptom-complex among female subjects with a low degree of courage (figure 4b2) is clearly seen in the age periods (from 11-12 to 20 years of age), when both graphs almost merge, and only in primary school age (9-11 years of age) and among students (21-26 years of age) their changes are somewhat divergent.

The authors of the article express their gratitude to B.Ch. Meskhi, the principal of DSTU, for the opportunity for conducting the study on the basis of the DSTU track-and-field athletic hall, to O.S. Mavropulo, the director of Institute of Physical Culture and Sports, for valuable advice in planning the study and recommendations for the formatting of the article.

\section{References}

1. L.A. Belozerova, E.A. Bragina, I.A. Semenova, I.A. Semikasheva, Theory and Practice of Physical Culture 3 (50), 15-17 (2018).

2. G.V. Rudenko, Yu.A. Dubrovskaya, I.V. Bobrov, Theory and Practice of Physical Culture 4 (49), 8-10 (2018).

3. Tina J. Kauh, Preventive Medicine 130, 105894 (2020).

4. S.G. Farris, E.K. Burr, M.M. Kibbey, A.M. Abrantes, A.M. DiBello, Mental Health and Physical Activity 19, 100346 (2020).

5. P.S. Tandon, Melanie Klein, B.E. Saelens, D.A. Christakis, Mental Health and Physical Activity 15, 17-21(2018).

6. A.A. Ivanova, N.V. Ryzhkin, I.A. Stepanova, E.A. Nurdygin, Physical education: education, training 2 (79), 53-56 (2019). 
7. A.A. Ivanova., O.Y Brovashova, Adaptation and development of the physical culture and sports complex of the GTO in accordance with changes in society, In the collection: Innovative transformations in the field of physical culture, sports and tourism. Collection of materials of the XXI All-Russian scientific and practical conference, 287-292(2018)

8. P.S. Tandon, Melanie Klein, B.E. Saelens, D.A. Christakis, Mental Health and Physical Activity 15, 17-21(2018)

9. Samira Rahimi, Maghsoud Peeri, Mohammad Ali Azarbayjani, Leila Anoosheh, Physiology \& Behavior 226, 113130 (2020).

10 T. Stepanova, R.M. Olkhovsky, E.A. Vashchenko, N.V. Ryzhkin, Theory and practice of physical culture 8, 98-99 (2018).

11 J.E. Simon,C.L. Docherty, Journal of Science and Medicine in Sport 21 (12), 12061209 (2018).

12. T. Wisbey-Roth, Journal of Science and Medicine in Sport 22 (1), 1 (2019).

13. B.S. Frank, A.C. Hackney, C.L. Battaglini, Troy Blackburn, Journal of Science and Medicine in Sport 22 (1), 29-34 (2019).

14. J. Luke Pryor, Riana R. Pryor, Lesley W. Vandermark, Elizabeth L. Journal of Science and Medicine in Sport 22 (1), 117-122 (2019).

15. M. Shestakov. S. Agafonov, Scientific notes of the University. P.F. Lesgaft 5(147), 199-203 (2017). 